\title{
Propagation des crues avec le Constrained Linear System (CLS) : Application en temps réel dans le système informatique de prévision
}

\author{
Marek Erlich \\ Laboratoire d'hydraulique de France (LHF)
}

\section{Description de la méthode}

L'algorithme de CLS calcule l'hydrogramme sortant $Q(t)$ comme une somme de réponses linéaires d'un certain nombre d'" entrées " hydrologiques. Il est fondé sur une théorie d'identification des systèmes à entrées multiples et une sortie.

Dans le cas général d'un système avec plusieurs entrées et plusieurs sorties:

$$
\begin{aligned}
\sum_{i=1}^{N} a_{i} \frac{\mathrm{d}^{\mathrm{i} Q} \mathrm{~d}}{d t^{i}} & \\
& =\Phi\left(\sum_{i=0}^{N-1} b_{i} \frac{d^{i} P}{d t^{i}}, \sum_{i=0}^{N-1} \sum_{j=0}^{N} c_{i} \frac{d^{i} P\left(t-t_{j}\right)}{d t^{i}}, t\right)
\end{aligned}
$$

$0 \leqslant t_{0}<t_{1}<\ldots<t_{j}<\ldots<t_{n}$

on distingue quatre types de systèmes suivant les caractéristiques de leurs paramètres :

(1) si les paramètres $a_{i}, b_{i}, c_{i}$ et $\Delta t_{j}=t_{j+1}-t_{j}$ sont constants, le système est linéaire et invariant dans le temps;

(2) si $a_{i}, b_{i}, c_{i}$ ou $\Delta t_{j}=\mathbf{f}_{n}(t)$, le système est linéaire et variant dans le temps;

(3) si $a_{i}, b_{i}, c_{i}$ ou $\Delta t_{j}=\mathbf{f}_{n} \quad$ le système est non linéaire et invariant dans le temps;

(4) si $a_{i}, b_{i}, c_{i}$ ou $\Delta t_{j}=\mathbf{f}_{n} \quad$ le système est non linéaire et variant dans le temps.

Le modèle $C L S$ décrit le système réduit avec entrées multiples et sortie unique (fig. 1) et, dans le cas général, on suppose qu'il représente un système hydrologique linéaire, invariant dans le temps avec des paramètres localisés. Il se compose de débits d'entrée $I_{j}(t)(j=1$, $2, \ldots, \ell)$, précipitations d'entrée $P_{i}(t)(i=1,2, \ldots, k)$ et du débit sortant $Q(t)$.

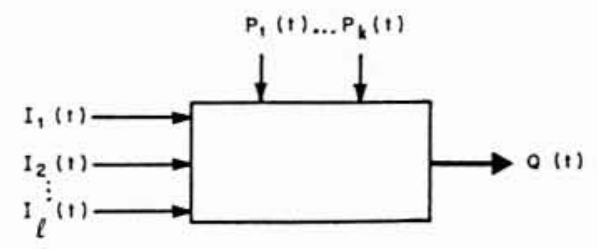

1.

\section{Propagation of floods with the constrained linear system (CLS).}

Real time application in the forecast information system

A model constructed with the CLS is a model derived from a systematic approach based on the evaluation of the transfer function between several hydrologic inputs and one output in the form of a linear relation. The article covers a general description of the model, taking into account of the linearities of an hydrological system, and the real time application (modelization of residues, degraded mode). Its comparison with other propagation methods is fully covered in this report.

\section{LA HOUILLE BLANCHE/N 5/6-1988}


La relation entre tous les éléments du système linéaire est donnée par :

$$
\mathbf{q}=H \mathbf{u}+\boldsymbol{\varepsilon}=\sum_{i=1}^{N} H_{i} \mathbf{u}_{\mathrm{i}}+\boldsymbol{\varepsilon}
$$

où :

$\mathbf{q}=[Q(1), \ldots, Q(m)]$ est le vecteur des débits sortants;

$H=\left[H_{1}, H_{2}, \ldots, H_{N}\right]$ est la matrice des entrées dont les éléments $H_{i}$ sont définis de la manière suivante :

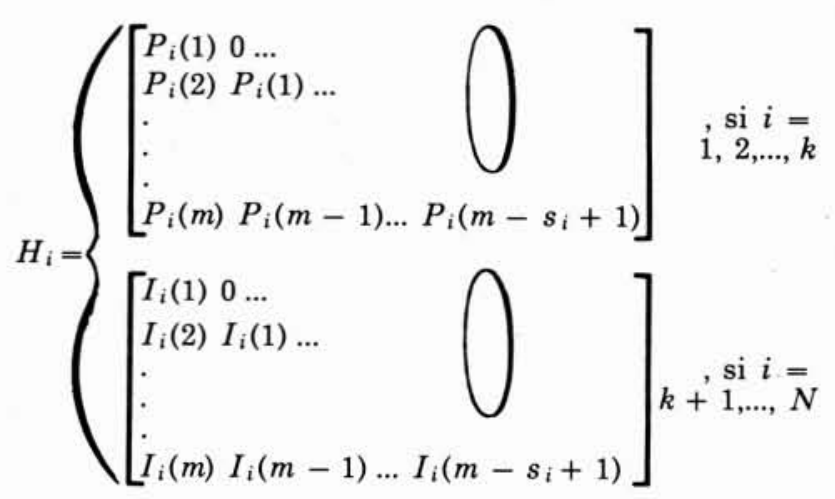

où :

$S_{i}$ signifie le nombre de paramètres d'une réponse unitaire de l'entrée $i$;

$\mathbf{u}=\left[\mathbf{u}_{1}, \ldots, \mathbf{u}_{N}\right]$ est le vecteur des réponses unitaires et chacun de ses éléments est défini comme:

$$
\mathbf{u}_{i}=\left[u_{i}(1), u_{i}(2), \ldots, u_{i}\left(s_{i}\right)\right],
$$

$i=1,2, \ldots, k, k+1, \ldots, N$;

$\varepsilon=[\varepsilon(1), \varepsilon(2), \ldots, \varepsilon(m)]$ est le vecteur des erreurs;

$N=k+\ell$ est le nombre total des entrées.

L'estimateur le plus efficace (BLUE) des fonctions de transfert du système (2) est celui qui minimise la fonctionnelle $([4,5])$ :

$$
\min \mathbf{J}(\boldsymbol{\varepsilon})=\frac{1}{2} \boldsymbol{\varepsilon}^{T} \boldsymbol{\varepsilon}=\frac{1}{2}(q-H \mathbf{u})^{T}(\mathbf{q}-H \mathbf{u})
$$

Le modèle (2) pourrait être étendu par l'introduction d'un terme autorégressif sur le débit sortant:

$$
\begin{aligned}
Q(t)= & \sum_{r=1}^{R} \alpha_{r} Q(t-r)+\sum_{j=1}^{\ell} \sum_{\tau=0}^{s(j)} h_{j}(\tau) \cdot I_{j}(t-\tau) \\
& \sum_{i=1}^{k} \sum_{\tau=0}^{T(i)} h_{i}(\tau) \cdot P_{i}(t-\tau)+\varepsilon(t)
\end{aligned}
$$

ici :

$\alpha_{r}$ sont les coefficients d'autorégression ( $r=1$, $2, \ldots, R)$

$R \quad$ est le retard maximal de la fonction d'autocorrélation sur la sortie

$t \quad$ est le pas de temps, $t=1,2, \ldots, m$

$h_{i}, h_{j}$ sont les poids de réponses unitaires pour les différentes entrées $(i=1,2, \ldots, k ; j=1,2, \ldots, \ell)$ $\tau \quad$ est l'indice de retard

$s(j) \quad$ est le retard maximal pour l'entrée $j$

$T(i)$ est le retard maximal pour l'entrée $i$

$\varepsilon(t) \quad$ est l'erreur résiduelle

Les entrées $I_{j}$ sont représentées par les hydrogrammes correspondants, tandis que les entrées $P_{i}$ sont représentées par les hyétogrammes, tous transformés ensuite en hydrogrammes de sortie $Q(t)$.

Le terme autorégressif représente l'influence du passé proche de la station elle-même, c'est-à-dire de l'endroit où le débit $Q(t)$ est calculé.

CLS est un modèle corrélatif qui trouve la solution unique de l'équation (1) avec (ou sans) un esnemble de contraintes de types différents que l'on peut attacher à chacune des entrées. Pour le système sans contraintes, on cherche d'abord le modèle d'auto-corrélation des débits sortants (si l'on opte pour un modèle avec terme autorégressif) et ensuite on utilise la méthode des moindres carrés (OLS) qui donne l'estimateur non biaisé de la fonction de transfert:

$$
\mathbf{u}=\left(H^{T} H\right)^{-1} H^{T} \mathbf{q}
$$

Dans le cas où l'on impose les contraintes :

$$
\begin{array}{ll}
\mathbf{u} \geqslant 0 & \text { contraintes de non négativité } \\
G \mathbf{u} \leqslant \mathbf{i} & \text { contraintes de continuité }
\end{array}
$$

où $G$ est une matrice de pertes entre les entrées et la sortie et i est un vecteur unité, il faut trouver l'estimateur linéaire contraint (CLS) du vecteur $\mathbf{u}$ :

$$
\min \mathbf{J}(\mathbf{u})=\frac{1}{2} \mathbf{u}^{\mathrm{T}} \mathbf{H}^{\mathrm{T}} \mathbf{H} \mathbf{u}-\mathbf{u}^{\mathrm{T}} \mathbf{H}^{\mathrm{T}} \mathbf{q}
$$

L'algorithme CLS permet de généraliser des contraintes imposées en remplaçant (7) par:

$$
G \mathbf{u} \leqslant \boldsymbol{\beta}
$$

où $\beta$ est un vecteur de constantes arbitraires.

La solution du problème $(8)$ sous contraintes $(6)$ et/ou (9) est trouvée par une approche de la méthode de programmation quadratique, qui utilise le gradient projeté pour résoudre le problème de minimisation si $\mathbf{J}(\mathbf{u})$ est convexe (TodinI et WaLLIS [3]).

La signification physique de la contrainte (6) est le sens unique d'écoulement (amont-aval), celle de la contrainte (7) est liée à la continuité du phénomène.

\section{Les algorithmes de désagrégation des en- trées}

Pour un certain nombre de problèmes non linéaires (mais toujours non invariants en temps), CLS dispose d'un algorithme de séparation de chacune des entrées en deux ou plusieurs segments, pour calculer les fonctions de transfert séparément.

Cette nécessité vient de la non homogénéité d'un système hydrologique, c'est-à-dire que des impulsions identiques peuvent conduire à une réponse différente.

La méthode de désagrégation est basée sur la partition de chaque entrée par des seuils arbitraires. On fait la distinction entre deux types d'entrée. 
Pour générer les pluies, TodinI et WALLIS [4] ont introduit dans CLS une fonction des conditions d'humidité antécédentes $(A P I)$ définie comme :

$A P I(t)= \begin{cases}0, & \text { si } t=1 \\ K_{t} * A P I(t-1)+P(t-1), & \text { si } t=2, \ldots, m\end{cases}$ où :

$$
K_{t}=\bar{K}+\delta \cdot \cos \left(\frac{2 \pi}{365}(t+\varphi)\right)
$$

$\bar{K}$ est une valeur moyenne de $K_{t}$

$\delta$ est une amplitude

$\varphi$ est une phase (en jours)

Ici, $K_{t}$ représente les variations périodiques de l'indice de précipitation antécédent et $P(t-1)$ représente la valeur de pluie au temps précédent.

Ensuite, si pendant une certaine période $L$ la pluie totale dépasse un seuil arbitraire $T$, on partage le vecteur d'entrée en deux ou trois éléments.

Pour les entrées sous la forme d'hydrogramme, l'algorithme prévoit l'application de la partition:

- soit par un seuil vertical sur une entrée donnée selon la valeur du gradient $d I / d t$,

- soit par un (ou deux) seuils horizontaux, selon la forme de crues.

Dans ce dernier cas, il s'agit de l'estimation de la fonction transfert par tranches des débits.

Avec deux seuils différents, CLS permet de décrire un système fortement non linéaire, correspondant souvent à la réalité hydrologique.

En raison de la convexité du problème (et l'existence d'une solution unique), il est important que chaque entrée ait au minimum une coordonnée différente de zéro.

\section{Tests de qualité du modèle CLS}

Les tests numériques classiques sont utilisés pour mesurer la qualité des modèles fournies par l'algorithme du CLS :

$-m$ : moyenne résiduelle

$$
m=\frac{1}{n} \sum_{t=1}^{n} \varepsilon(t)
$$

où $n$ est un nombre des débits considérés,

- $S$ : écart-type de la moyenne résiduelle

$S=\sqrt{\frac{\sum_{t=1}^{n}(\varepsilon(t)-m)^{2}}{n-1}}$

$-R^{2}:$ coefficient de détermination

$$
R^{2}=1-\frac{\sum_{t=1}^{n} \varepsilon^{2}(t)-n \cdot m^{2}}{\sum_{t=1}^{n} Q^{2}(t)-n \cdot Q^{2}}
$$

où :

$$
Q=\frac{1}{n} \sum_{t=1}^{n} Q(t)
$$

est la moyenne de débits observés

- $E_{1}$ : différence, en pourcentage, entre les pointes de crues observée et calculée

$$
E_{1}=\frac{\hat{Q}_{\max }-Q_{\max }}{Q_{\max }} \cdot 100 \%
$$

- $E_{2}$ : erreur de décalage entre deux pointes

$$
E_{2}=t_{Q_{\max }}-t_{Q_{\max }}
$$

où :

$t_{Q_{\max }}$ est l'instant où l'on observe le débit de pointe $Q_{\max }$ et $t_{Q_{\max }}$ correspond à l'instant de maximum de débit calculé $\hat{Q}_{\max }$.

\section{Application dans le cadre du Niger}

Un exemple d'application du CLS dans le projet HYDRONIGER [6] explique la simplicité du réglage d'une fonction de transfert. Dans la phase de construction du SIP pour un tronçon du Niger moyen (3 stations d'entrée: Tossaye, A1 Congui et Garbe-Kourou, station de prévision à Niamey), on a étudié 4 modèles de CLS.

\section{Modèle M1}

Sans aucune contrainte, il n'est qu'un simple modèle corrélatif qui n'apporte pas d'informations utiles sur la fonction de transfert. De plus, l'optimisation d'un système sans contraintes donne une solution libre, c'est-à-dire que les valeurs des coefficients de la réponse unitaire sont successiblement positives et négatives sans signification physique.

\section{Modèle M2}

Dans le modèle M2, on impose seulement des contraintes positives. Les valeurs des coefficients de réponse unitaires, propres à chaque entrée, n'ont pas de sens physique en raison de la bonne corrélation entre les débits des stations amont.

\section{Modèle M3}

Le modèle $\mathrm{M} 3$, avec deux types de contraintes (non négatives et de continuité) fournit une crue sous-estimée du point de vue du bilan de masse, mais correcte du point de vue de la modélisation mathématique.

\section{Modèle M4}

Le modèle de type M4 est un modèle M2 avec un option supplémentaire de modélisation autorégressive des débits sortants. En effet, tout le poids d'estimation passe à la fonction d'autocorrélation des débits à Niamey 


\begin{tabular}{|c|c|c|c|c|c|c|c|c|c|c|}
\hline \multirow{2}{*}{$\begin{array}{l}\text { Type de } \\
\text { modèle } \\
\text { de CLS }\end{array}$} & \multicolumn{5}{|c|}{$\begin{array}{l}\text { Période de réglage } \\
\text { ( } 1 \text { aout - } 30 \text { avril 1967-1976) }\end{array}$} & \multicolumn{5}{|c|}{$\begin{array}{c}\text { Période de test } \\
(1 \text { août }-30 \text { avr11 1977-1978) }\end{array}$} \\
\hline & $\mathrm{m}$ & S & $R^{2}$ & $\mathrm{E}_{1}$ & $\mathrm{E}_{2}$ & $\mathrm{t} \mathrm{n}$ & $\mathrm{s}$ & $R^{2}$ & $\mathrm{E}_{1}$ & $\mathrm{E}_{2}$ \\
\hline M1 & -30.36 & 290.76 & 0.9797 & 10.23 & -2.0 & -13.07 & 209.39 & 0.9875 & 9.32 & 2.0 \\
\hline M2 & -21.04 & 366.35 & 0.9677 & 5.03 & 0.0 & 7.69 & 243.45 & 0.9830 & 7.36 & 2.0 \\
\hline Y3 & -24.17 & 415.99 & 0.9585 & 1.02 & 0.0 & -70.26 & 277.64 & 0.9779 & -1.025 & 4.0 \\
\hline M4 & 56.77 & 1641.80 & 0.3538 & -21.59 & 15.0 & 1.12 & 38.107 & 0.9996 & 1.118 & 1.0 \\
\hline
\end{tabular}

(traités comme une entrée) et les débits des stations amont sont utilisés comme une correction, donc les valeurs des coefficients des vecteurs unitaires ne correspondent pas à la réalité physique.

Le tableau ci-dessus représente les valeurs de ces estimateurs statistiques calculés en réglage et en simulation pour les quatre modèles CLS du tronçon du Niger pris comme exemple.

Le SIP, étant un outil de prévision complet (Erlich et Sauvaget [2]), permet d'utiliser plusieurs modèles réglés par des méthodes différentes et de comparer leur fonctionnement dans des scénarios de prévisions.

Pour comparer les performances du modèle CLS intégré dans le cadre du scénario de prévision du SIP avec le modèle Muskingum du même tronçon (fig. 2 à 5), les simulations sur 10 et 20 ans ont été effectuées (Chéné et Erlich [1]). Les deux méthodes ont produit des résultats comparables dans le sens des tests de qualité (11)-(15).

\section{Extrapolation aux stations amont}

Pour pouvoir faire de la prévision par le CLS ou Muskingum, il faut étendre les données d'observation aux stations entrée au-delà du temps d'actualisation $t_{\text {act }}$. Pour déterminer les paramètres des lois d'extrapolation, on utilise une procédure ARIMA aux débits observés. Il s'agit d'appliquer à chaque station à extrapoler un processus autorégressif d'ordre 2 sur les débits centrés par rapport au débit interannuel à la date donnée :

$$
\begin{aligned}
Q(t)-M(t)=a_{1} \cdot(Q( & t-1)-M(t-1)) \\
& +a_{2} \cdot(Q(t-2)-M(t-2))
\end{aligned}
$$

avec :

$Q(t)=$ débit à la station à l'instant extrapolé $t$,

$M(t)=$ débit interannuel à la même date,

$a_{i} \quad=$ coefficient de l'autorégression avec $i=1,2$.

Au début de l'extrapolation, quand $t$ est proche de $t_{\text {act }}$, le débit $Q(t)$ est surtout conditionné par le débit antérieur $Q(t-1)$ et le gradient de la crue $Q(t-1)-Q(t-2)$. A mesure que l'extrapolation s'éloigne dans le temps de l'instant $t_{\text {act }}$, elle est de plus en plus influencée par la forme de la crue moyenne.

\section{Comblement des lacunes et mode dégradé du SIP}

Pour pouvoir continuer le déroulement de la prévision dans le cas d'une défaillance de transmission ou d'une panne de la balise ARGOS, des modèles spéciaux dits de comblement ont été stockés dans la banque des modèles. Ces cellules de remplacement assurent le fonctionnement du système en mode dégradé.

Pour avoir une liaison acceptable, tenir compte de l'influence de la saison sur les écoulements et ajuster les formules dans une plage de débits limitée, les cellules de comblement sont des régressions multiples saisonnières, avec une ou deux stations voisines. Pour combler une station, on peut avoir plusieurs modèles concurrentiels.

\section{Conclusion}

Un modèle construit avec le CLS est basé sur l'estimation de la fonction transfert, dont les paramètres sont déterminés d'une manière optimale, en tenant compte de certaines contraintes, par exemple la continuité entre les volumes entrants et sortants d'un bassin versant. L'algorithme du CLS calcule le débit aval comme étant la somme des réponses linéaires de plusieurs stations amont. Son application, dans le cadre du projet HYDRONIGER, a permis d'intégrer les modèles CLS à l'ensemble des méthodes de prévision utilisées dans le SIP.
Adresse de l'auteur

Monsieur Marek Erlich

LHF

6, rue de Lorraine

38130 Echirolles

Tél. : (16) 76.33.42.08 


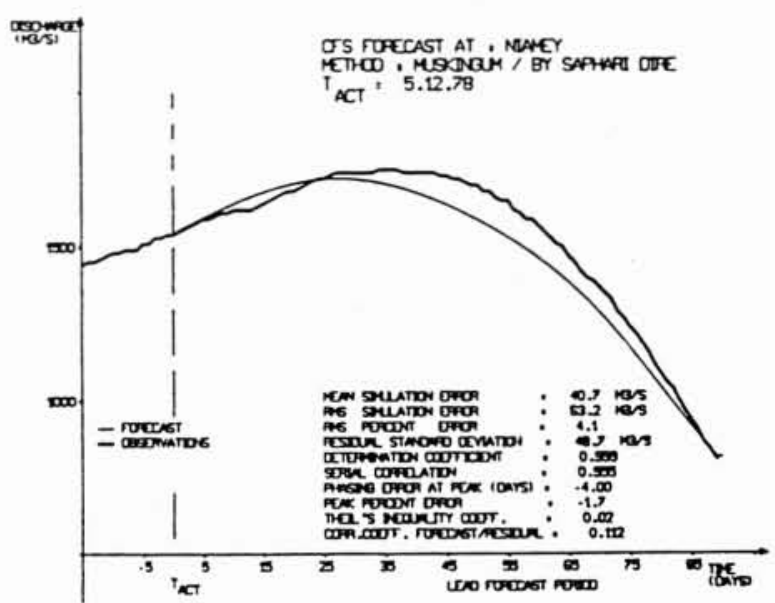

2.

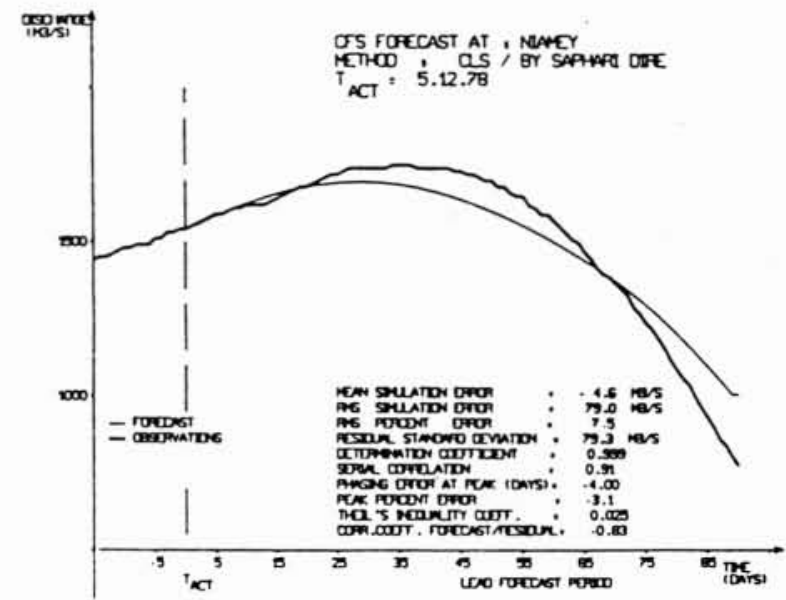

4.

\section{Références}

[1] ChÉnE J.M. and ERLICH M. - On combining the classic propagation models with a multivariate seasonal multimodel competition system : the application for real-time forecasting of the Niger River Basin discharges, Proc. of the 4th Intern. Symposium on Multivariate Analysis of Hydrologic Processes, July 15-17, Colorado State Univers., Fort Collins, 1985.

[2] Erlich M., Sauvaget P. - Computerised Forecasting System : a general software product for interactive manag. ment of hydrology data banks and real-time river discharge forecasting, Proc. of Hydrosoft'86, Southampton, Sept. 9-12, U.K., Springer-Verlag, 1986, pp. 333-355.

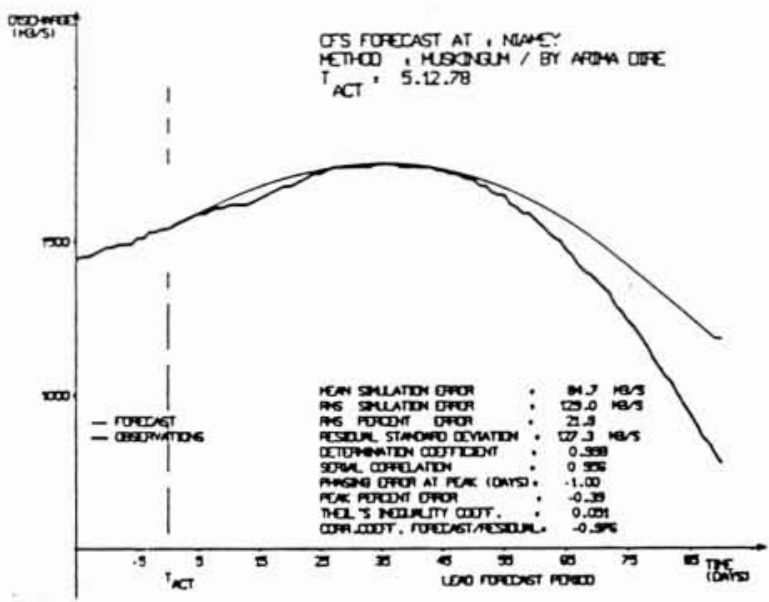

3.

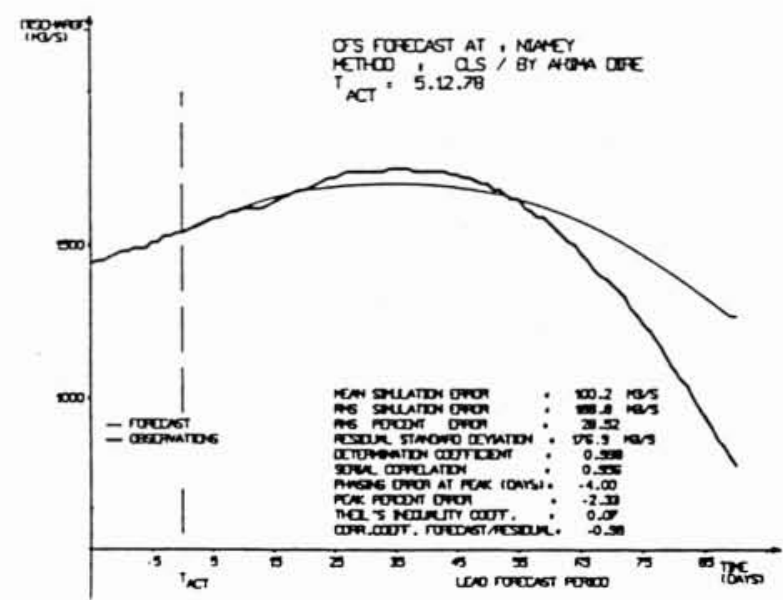

5.

[3] TodinI E. - Using a desk-top computer for an on-line flood warning system, IBM Journal of Research and Development, vol. 22 , No 5,1978 , pp. 464-471.

[4] TodinI E. and WALLIS J.R. - Using CLS for daily of longer period rainfall-runoff modelling, in: Mathematical models for surface hydrology, Ed. J. Wiley \& Sons, NY, 1977, pp. 149-168.

[5] YAZICIGIL H., RAO A. - Investigation of daily flow forecasting models, Journal of Water Resources Planning \& Manageinent, ASCE, vol. 108, No WR1, March 1982, pp. $67-85$.

[6] Système informatique de prévision (SIP), projet Hydroniger, RAF/79/055, OMM/Sogreah, septembre 1984. 
M. DUJARDIN : Je voudrais faire deux commentaires. Tout d'abord le CLS est facile à manier, mais on a moins de facilité à comprendre le sens physique des paramètres que dans un modèle Muskingum : la démarche est plus mathématique donc plus systématique mais aussi plus aveugle. Deuxièmement il est apparu qu'un classement comparatif des méthodes est très difficile à faire. Dans le cas du système de prévision du Niger, les performances respectives des méthodes en concurrence, CLS, Muskingum et Sphari sont très variables selon les crues, les délais et les points de prévision considérés.

M. DUBAND : Vous utilisez un modèle ARIMA et ARMA en prévision, mais je pense que ce sont des modèles faibles en matière de prévision hydrologique au-delà du premier pas de temps. Savez-vous si le modèle CLS est opérationnel actuellement sur le Niger et sur le Beijing ?

M. ERLICH : Sur le Beijing, je n'en sais rien, mais, sur les rivières Han et Huai en Chine, une société italienne a installé, dans le cadre d'un système opérationnel de prévision, un modèle CLS; le pas de temps d'acquisition est de une heure, le pas de temps de prévision est de trois, six et vingt-quatre heures. Sur le Niger, le système SIP est installé depuis 1984 et utilise un certain nombre de schénarios basés sur des modèles différents. Certains scénarios, qui résultent de l'étude présentée ici, sont standard, et leur utilisation est conseillée; d'autres sont possibles. Le choix final revient à l'utilisateur, c'est-à-dire à l'Autorité du Bassin du Niger. Il y a certainement des améliorations à apporter au niveau de la prévision des débits du fleuve. L'Organisation Météorologique Mondiale envisage d'équiper des stations " pluvio " en balises de transmission et d'inclure dans le processus de prévision les modèles type pluie-débit. Il y a peut-être encore un certain chemin à faire.

M. THIRRIOT: J'ai deux questions, l'une au mathématicien, l'autre à l'Africain. Qu'en est-il sur la convolation non linéaire suite aux travaux d'Amarocho sur ce sujet ? Avez-vous une idée du temps caractéristique de la montée de crue dans le fleuve Niger, à Niamey par exemple?

M. ERLICH : L'application du modèle non linéaire a été très à la mode dans les années 70 , mais elle est très difficile numériquement, notamment en ce qui concerne l'estimation des noyaux dans un système orthonormal. Dans les publications récentes, les recherches sont orientées plutôt vers l'utilisation des multimodèles linéaires.

Concernant votre autre question, la montée de crue du Niger varie selon l'endroit; elle est très, très lente à Niamey, (le Niger est un véritable lac à la sortie du delta inférieur) où la durée moyenne est de l'ordre de 10 mois. Dans la partie amont (à Kourlikoro, par exemple), le temps de la montée et la durée totale sont respectivement de l'ordre de 3 et de 6 mois. A cause des conditions hydrologiques du Niger, il faut que l'utilisateur fasse appel à des méthodes et des techniques de modélisation différentes.

M. Moll: N'aurait-il pas été possible d'utiliser un filtre de Kalman dans le cas du Niger ?

M. ERLICH: Les essais que nous avons effectués avec un filtre de Kalman ont donné des résultats instables pour les prévisions à long terme; comme nous devions fournir un système opérationnel avec des prévisions à 90 jours, il n'était pas possible d'inclure un filtre de Kalman.

D'un autre côté, dans certaines stations on pourrait utiliser avec profit des prévisions à court terme, par exemple pour les stations en amont des barrages, dans ce cas, je pense que l'on pourrait utiliser en toute confiance un filtre de Kalman. 